\title{
Jean-Marie Legay, le scientifique, l'humaniste et le novateur
}

\author{
Alain Pavé \\ Biométricien, Université Claude-Bernard Lyon 1, UMR5558 Laboratoire de biométrie et biologie évolutive, 69622 Villeurbanne, \\ France
}

Jean Marie Legay a commencé sa carrière de scientifique en 1946. Ingénieur diplômé de l'Institut national d'agronomie, il a été employé comme stagiaire au service botanique et agronomique de Tunisie ${ }^{1}$. Puis ce fut $1^{\prime}$ Inra jusqu'en 1962, année de sa venue à la faculté des sciences de Lyon. Pendant cette période, pré-lyonnaise, il exerçait son métier de jeune chercheur à la station de Saint-Christollès-Alès en étudiant son modèle biologique favori : le ver à soie. Ce travail, encadré et jugé par de grands noms, Pierre-Paul Grassé et Georges Teissier, lui a permis de soutenir une thèse d'État, où la biométrie était déjà très présente. En 1962, Jean-Marie Legay a été sollicité par Victor Nigon, alors professeur de biologie et de génétique à la faculté des sciences de Lyon, pour créer un laboratoire de biométrie au sein de ce qui allait devenir la section de biologie générale et appliquée. Cette section a été l'un des premiers laboratoires associés au CNRS en 1966 (LA 72).

\section{Un universitaire soucieux d'universalité}

Sa carrière a été riche tant sur le plan scientifique qu'institutionnel. L'impressionnante liste de ses publications (articles et ouvrages) en fait foi. Elle témoigne non seulement d'une science très pointue mais surtout d'un grand éclectisme. Il a aussi consacré une partie importante de son temps à l'écriture d'ouvrages didactiques. Tâche essentielle pour un universitaire, bien que, dans nos disciplines, la pression pour multiplier les articles ne laisse que peu de loisir pour rédiger des livres. Et pourtant, ce sont eux qui, lorsqu'ils sont bons, restent dans la mémoire et s'inscrivent dans le long terme. On connaît

\footnotetext{
Auteur correspondant : alain.pave@univ-lyon1.fr

1 Voir aussi, dans ce numéro et le précédent, les autres hommages à Jean-Marie Legay.
}

également ses réalisations : le département de biologie appliquée de l'IUT, le laboratoire de biométrie à l'Université Claude-Bernard (Lyon 1), un institut interdisciplinaire (l'Institut d'analyse des systèmes biologiques et socioéconomiques) et un DEA (Analyse et modélisation des systèmes biologiques). Il a exercé diverses responsabilités, en particulier au CNRS, comme directeur scientifique adjoint au département des sciences de la vie, et il a obtenu plusieurs distinctions dont le prestigieux, à l'époque, prix Lénine de la science.

Grâce à son apport, l'école lyonnaise de biométrie est parfaitement identifiée et connue pour son originalité. Elle constitue toujours "l'âme » du Laboratoire de biométrie et biologie évolutive (LBBE, UMR5558), stade actuel de l'évolution du laboratoire d'origine ${ }^{2}$, et imprègne fortement une école doctorale. C'est le résultat d'un travail de longue haleine d'enseignements méthodologiques dans les filières biologiques et écologiques de la faculté des sciences puis de l'Université ClaudeBernard Lyon 1, couplé à une recherche scientifique de pointe. Effort sans lequel rien n'aurait pu être construit. Suivant cette logique, l'émergence d'une filière de biomathématiques, après plus de trente ans de persévérance, place l'Université de Lyon dans une position très originale, de plus en plus imitée. Au début des années 2000, c'est aussi à partir de cette expérience que des membres du laboratoire ont participé à la création d'une

\footnotetext{
2 Au début, ce laboratoire comptait une dizaine de personnes ; aujourd'hui, il y en a plus de 200. Ont été chercheurs dans ce laboratoire : deux membres de l'Académie des sciences, un membre del'Académie des technologies et correspondant de l'Académie d'agriculture, un PDG d'un grand institut de recherche (l'Inra). Plusieurs chercheurs et enseignantschercheurs ont été médaillés du CNRS (bronze et argent). Il compte aussi des membres de l'Institut universitaire de France. Nombreux sont ceux qui ont été formés à cette école lyonnaise de biométrie et exercent ailleurs leur passionnant métier.
} 
spécialité «bio-informatique et modélisation » à l'Insa de Lyon avec le ferme soutien de son directeur de l'époque, Joël Rochat. Les ingénieurs ayant suivi ce cursus sont, depuis la première promotion, très sollicités. De fait, Jean-Marie Legay a été visionnaire avant l'heure. Les interactions biologie-mathématiques et biologieinformatique sont devenues des évidences. Ne serait-ce que sur ce plan, son bilan est déjà prodigieux.

Sous l'incitation permanente de Jean-Marie Legay, un travail de longue haleine a également été mené dans le laboratoire de Lyon pour maintenir la dualité biologiebiométrie. Ce travail ancre ainsi la biométrie dans le concret de la recherche biologique et écologique. Il assure aux biologistes un support méthodologique de proximité quasiment sans équivalent. Il s'inscrit dans le long terme et a été servi par une remarquable clairvoyance dans les choix de Jean-Marie Legay, poursuivis et renforcés par ses successeurs, l'auteur de cette note en premier. Le LBBE en tire tous les bénéfices aujourd'hui.

Bien entendu, on ne peut parler de Jean-Marie Legay sans évoquer l'interdisciplinarité. Il a poussé la logique jusqu'à revendiquer une "indisciplinarité », en s'attaquant à l'édifice qui continue à peser lourdement sur la science, sans pour autant nier l'efficacité de l'approche disciplinaire. Nombre de ses écrits en témoignent ainsi que son engagement dans Natures Sciences Sociétés. Il a été l'un de ceux, aussi, qui " passent les frontières » sans en demander la permission.

Toujours en suivant cette logique, Jean-Marie Legay a créél'Institut d'analyse des systèmes biologiques et socioéconomiques (IASBSE), au sein de 1'Université ClaudeBernard. Il en a assuréla direction pendant deux mandats. Dix ans après sa création, on murmurait que c'était sans doute l'institut de l'Université qui marchait le mieux... Hélas, cette structure innovante n'a pas résisté à une réforme, qui a, une fois de plus, organisé l'Université selon des schémas disciplinaires.

Son passage au CNRS a été marqué par la juste reconnaissance - dans la rigueur et la qualité scientifiques des recherches écologiques, trop souvent négligées par le passé. Jean-Marie Legay a su convaincre les directeurs du département des sciences de la vie avec qui il travaillait, et, parallèlement, il a su mobiliser la communauté scientifique de l'écologie en lui imposant l'impératif de qualité. Cet impératif est le meilleur argument du CNRS, la meilleure caractéristique et la meilleure arme d'une écologie française en pleine évolution. Cette initiative a été très profitable pour soutenir les recherches sur l'environnement au CNRS et ailleurs.

Enfin, je ne peux résister au plaisir de souligner son éclectisme, la véritable qualification de savant qu'on pouvait lui donner. Ainsi, on trouve dans ses travaux des contributions à la physiologie des insectes et la sériciculture, évidemment à la biométrie, à l'écologie et à la biologie des populations animale, végétale et humaine, à la bio-informatique, à la morphométrie, à la modélisation, à l'écologie urbaine dont il a été l'un des promoteurs sinon l'un des pionniers, à l'épistémologie, et j'en oublie. Il a su dispenser son savoir et sa pensée ; il nous a souvent étonnés et stimulés; il nous a aussi appris à confectionner quelques entremets scientifiques.

\section{De provocations en paradoxes}

La lecture d'une liste de publications, ou même de plusieurs d'entre elles, la connaissance de ses réalisations, de ses responsabilités et de ses distinctions, ne renseignent pas assez quant à la richesse de la personne, quant à ses qualités humaines et scientifiques. Je prends donc la liberté de sortir des sentiers battus, mais néanmoins plein d'ornières, du panégyrique et de prendre une anecdote et quelques faits qui m'ont plus particulièrement marqué et qui me semblent les plus révélateurs d'une personne d'exception.

L'art de manipuler le paradoxe et le raisonnement provocateur le caractérisait. Peu après 1968, c'était l'époque où les premières machines électroniques commençaient à envahir le labo. Un lundi matin, nous commentions, avec un proche collègue, Jean-Luc Chassé, la fuite d'eau du week-end provenant d'un étage supérieur. Ce type d'événement nous faisait froid dans le dos, car nous avions peur pour nos chères et coûteuses machines. À ce propos donc, nous informions Jean-Marie Legay de l'origine de l'incident, à savoir, au cours du week-end, la rupture d'un tuyau de raccordement entre une trompe à vide et un robinet. Très calmement, il nous a rétorqué que les gens n'étaient pas raisonnables parce que, et je le cite de mémoire : "Comme chacun sait, tout le monde prend son bain le vendredi soir à 17 heures. Il y a donc une baisse de pression sur le réseau précisément à l'instant où le biologiste met en marche sa trompe à vide avant de partir pour le week-end. »

On imagine la suite... De fait, il illustrait ainsi, d'une part, le concept fondamental de non-stationnarité d'un phénomène et, d'autre part, l'importance de l'approche interdisciplinaire des problèmes: en l'occurrence, les causes sociologiques de l'incident! C'était surtout une provocation pour déclencher des réactions.

Parmi beaucoup d'autres choses, il faut rappeler, qu'il avait une grande expérience $\mathrm{du}$ fait biologique, par exemple, en proposant une réponse à l'une des questions qui nous tracasse tous depuis notre plus tendre enfance : mais enfin... pourquoi deux sexes?

Derrière la façade sérieuse et parfois austère, JeanMarie Legay avait aussi beaucoup d'humour. 


\section{Au-delà de l'homme de science, l'homme de l'amitié}

Je dois enfin évoquer nos relations, en impliquant aussi celle qui fut sans doute sa plus proche collaboratrice, son assistante de direction : Marie-José Pavé, avec qui j'ai vécu 30 merveilleuses années. Ensemble, ils étaient redoutables. De véritables complices, pour la bonne cause, notre cause collective. Beaucoup plus, nous étions liés au point que Jean-Marie et Simone Legay furent les premiers à voir Marc dès le lendemain de sa naissance, un 27 octobre 1969. Puis ce furent de nombreuses visites à Saint-Just Chaleyssin, la chaleur des accueils de Simone et de Jean-Marie et les longues discussions des après-midi et des soirées d'été.
Ayant le profond désir de le revoir après mes « aventures amazoniennes ", je ne me rendais pas compte de l'urgence. Un regret sans doute, mais aussi le sentiment d'un honneur, celui de l'avoir connu et d'avoir profité de ses enseignements et de son amitié. D'une certaine façon, pour moi, comme pour beaucoup, il était porteur d'éternité. L'idée de sa disparition nous était étrangère. Physiquement, il n'est plus là, mais il nous laisse un précieux héritage. Jean-Marie Legay n'était pas préoccupé par les indices quantitatifs d'évaluation de la science. Il n'avait pour souci de multiplier les publications de résultats ponctuels. En revanche, il nous a livré des textes de réfèrence dont on parler a longtemps. 